\title{
COPYRIGHT AND PARATEXT IN COMPUTER GAMING*
}

\author{
DAN L. BURK \\ University of California, Irvine School of Law \\ 3500 Berkeley Place \\ Irvine, CA 92617-8000 \\ USA
}

\begin{abstract}
Adoption of information technologies is dependent upon the availability of information to be channeled via such technologies. On-line multiplayer role-playing games have become an important social and business feature of the Internet. The virtual worlds that game players inhabit now encompass population counts and economic activity greater than that of many nations in the physical world. The activity of players participating in an on-line game community is closely tied to paratexts that may include magazines, websites, and even devices that lie outside the formal boundaries of the game, but which are intimately bound up in the transmission of knowledge and culture surrounding the game. In this paper, I examine the legal structures that foster or inhibit particular gaming paratexts. Such laws are in some senses extrinsic to the "magic circle" of the game, but these external rules are deployed as constraints to enforce the internal logic of the game. Typically this occurs in cases where violation of the game's internal parameters would affect the owner's external marketing or business control. Commercial game developers may use copyright and related anti-circumvention laws to enforce preferred readings of the game, largely by dominating the paratexts associated with their product. Examining the control of paratexts yields important insights into the logic of legal rubrics governing on-line gaming.
\end{abstract}

\section{Introduction}

Gaming, like ethics, and like law, is about the rules: who makes the rules, who breaks the rules, how and when and why the rules are enforced. Until recently, breaking the rules of an entertaining game was for the most part merely impolite, perhaps entailing a violation of whatever ethical rules that encompassed the game's rules. In certain high-stakes games, marking the cards or loading the dice might even be a legal violation. But it was hardly a game

*Forthcoming in Emerging Ethical Issues of Life in Virtual Worlds (Charles Wankel \& Sean Malleck eds., Information Age Publishing). 
publisher's concern if some players learned to cheat at Monopoly or Risk, or if the players altered the rules to suit themselves. Neither did society worry whether publishers had set the right rules for the game, or what the effects might be if the publisher changed the rules.

Pervasive digital media have set the stakes higher for making, breaking, and enforcing the rules. Computer games require a higher investment on the part of developers, who expect a higher return. Player misdeeds may threaten that return, prompting developers to seek broader control over the rules. Online multi-player gaming offers a particular challenge to game developers due to the large number of players interacting in real time. To maintain the integrity of their games and their business models, the providers of computer games are increasingly turning to legal claims, particularly to copyright, to police the rules of their games.

In this chapter I discuss the implications that such legal strategies have for the interaction between developers and players, including issues of ownership, control, and autonomy. I will be particularly concerned with the effects of copyright on two key aspects of gaming: first, the impact of copyright on player's gaming capital, and second, copyright's effect on the production and interpretation of paratexts. I begin with an overview of the concepts of gaming capital and paratext as those terms have been applied to social analysis of computer gaming. I then move to a discussion of the copyright doctrines concerning substantial similarity and derivative works, their application to paratexts and their implications for gaming capital. I argue that the copyright regime confers on game publishers broad discretion to control not only the games that they publish, but also the texts surrounding and supporting the game proper. This in turn raises concerns regarding the control copyright confers over the meaning of texts, and the balance of expression and autonomy available to players engaging with gaming texts.

\section{Gaming Capital and Paratexts}

Consalvo identifies two key concepts that help to define the motivations and gaming behavior of on-line players. The first of these is that of gaming capital. (Consalvo, 2007) The concept of gaming capital incorporates a social aspect of game enjoyment that extends beyond the personal enjoyment derived from game participation. In many games, whether sports play, board games, games of chance, or others, players will derive enjoyment from the overcoming challenges presented the game, and from developing personal mastery over the nuances of the game. This level of enjoyment may then be enhanced by demonstrating game skill for spectators, or sharing tips with other players, and perhaps eventually becoming recognized as an expert or maven in the of the particular game. The recognition and acclaim that goes with expert status becomes an additional source of reward, encouraging not only development of 
skill in the game, but collection of information and expertise about the game. Players may seek to improve their social status among gamers by accumulating such gaming capital.

Gaming capital is not unique to computer gaming, nor to on-line multiplayer games; certainly it accrues in many other types of games. Game enthusiasts can readily draw on repositories of gaming lore that abound in diverse media. There are a wide variety of newsletters, fan groups, websites, and "how-to" books where experts pass knowledge on to less experienced players. Newspapers devote entire sections to reports, commentary, discussion, and statistics about sporting events. Even classic stand-alone video games include "best score" screen where the names or initials of accomplished players can be displayed to subsequent players.

But accrual of gaming capital is, if not unique to massive multiplayer games, at least distinctive in such games. In addition to repositories of gaming capital in texts that comment upon the game, the game itself provides a forum for accruing and expending gaming capital. Knowledge sharing, skill enhancement, game performance, and reputation development occur in real time during player interaction, with a potentially broad and diverse audience of recipients. Skilled and knowledgeable players become known not merely to fans or onlookers, but to a host of other participants in the course of play. Gaming capital becomes an integral feature of the network of social interactions within the game, as well as between aficionados outside the game.

From the standpoint of game developers, gaming capital is something of a mixed blessing. Accrual of gaming capital enhances the value and enjoyment of the game, driving adoption of the game, participation in the game, and ultimately increasing revenue under whatever business model is associated with the game. In the case of multiplayer computer games, where social interaction is an important feature of the gaming experience, reputational capital may accrue in real time as players interact; this immediate gratification is an important component of the players' experience. As a consequence, game developers will frequently rely implicitly or explicitly on this dimension of gaming in their game design, and in their business plans, to promote the popularity of their products. Not only will features of the game be calibrated to allow, even facilitate the accrual of gaming capital, but these features may be harnessed to assure the game's profitability.

Although the business model of the game may depend upon gaming capital, not all methods of accumulating gaming capital will be congruent with that model. Players may accumulate knowledge, not merely of the rules and skills and lore of the game, but regarding the technical structure of the game, allowing them to subvert or alter the computer code that governs play. Technically sophisticated players may hack the game for a variety of reason: to advance with less effort, out of spite or curiosity, even to accumulate virtual 
objects that may be marketed for real world profit. Perhaps more importantly, gaming capital may be accumulated by sharing scripts that allow other, less sophisticated players to circumvent the game code. At a somewhat less intrusive level, when players acquire or display their gaming acumen through the publication of web sites, fanzines, guides and manuals, they may interfere with marketing in peripheral markets that the game developer would prefer to capture. For example, players may develop and publish advice and "how to" manuals that the publisher would prefer to supply directly to players. Such scripts, manuals, guides and web publications lead us from the concept of gaming capital to a second key concept.

\subsection{THE ROLE OF PARATEXTS}

The second related and key concept identified by Consalvo is that of paratext. (Consalvo, 2007) The concept is drawn initially from Girard Ginette, who used it to designate texts that modify or enhance the reader's interaction with a primary text. In its original conception, the paratext category referred to elements of a published book such as the preface, index, dust jacket summaries, and even book reviews that are contributed by editors, publishers, and reviewers rather than by the primary text author. Such texts are connected to, but distinct from the text proper. They border primary text, mediating the understanding and the interactions of a reader approaching the primary text. Frequently they serve to define the text that they reference.

More recent commentators have interpreted, and perhaps re-interpreted the term broadly in accordance with current understandings of new media. (Lunenfeld, 1999) The concept of paratext is particularly salient in the context of digital media because of the malleability of texts based upon bits. This inherent flexibility allows the reader to re-formulate the text, combining it with other texts, literally and figuratively linking it to other texts, reorganizing and reconfiguring its content, porting the content between formats. Such user interactions "recode," the text, both in the sense of re-defining the meaning of a text and literally in the sense of adding or altering the code upon which digital texts are founded. The production of new texts, particularly paratexts, is characteristic of such recoding. Paratexts exist on a continuum of interaction with the primary text; some paratexts will interact with the initial text from a distance, encouraging new reader interactions with the primary text; others will create new readings by directly modifying or interacting with the primary texts. In print media, such conceptual interaction was reflected in the physical situation of texts: forewords and dust jackets were physically associated with the primary text of books, while reviews and commentary were located in separate print objects. But the fan web sites, fan fiction, discussion boards and blogs that mediate games and other digital texts are increasingly technically linked to the primary text so as to become nearly indistinguishable. 
Consalvo follows this construction of paratexts, expanding the class of primary texts to include computer games as a type of text. (Consalvo, 2007) Her definition of paratexts follows this expanded understanding, to include not only liminal texts physically attached to a primary text, but also separate texts, often in other media, that are dependent upon the character of the primary text. As used by Consalvo, the term encompasses a wide variety of gaming reviews, magazines, and similar materials that surround and draw upon the game text repositories of gaming capital that lie adjacent to the primary text. These texts also mediate the experience of the player, providing information about the game, creating expectations, offering strategies and advice. Even certain technical artifacts that might not be immediately recognized as texts may function as paratexts, speeding or slowing or altering game play by interacting with the game's software code.

\subsection{PLAYING AND CHEATING}

Consalvo is interested in these concepts as they illuminate the definition of cheating in computer game play. Cheating transgresses the set of normative and even legal practices acceptable to the game. The rules of play may be explicit or implicit, express or implied. Some norm of play must be violated for a practice to be defined as cheating, but different actors in the gaming world may have different perspectives about the norms at issue, and what violates those norms. Some activities may be considered cheating by other players; some practices may be considered cheating by game developers. These two sets may overlap, even to a large degree, but are not necessarily fully contiguous. Players may regard as innocuous some activity to which the game developer would object, and the developer may be unconcerned with types of play that players find objectionable.

Indeed, players may be unconcerned, and even contemptuous of restrictions imposed by the developer but perceived to be directed to the profit or benefit of the owner. The two perspectives are intertwined to the extent that the proliferation of behavior that players find objectionable will drive players from the game, adversely affecting the playability, popularity, and ultimately profitability of the game. And of course too much activity that circumvents or subverts the developers business model will cause the developer to abandon the game as unprofitable, leaving the players without the resource for their activities.

Depending on the game and the community associated with the game, behaviors defined as cheating may range from obtaining advanced knowledge of the game's features to unauthorized alteration of the game's underlying code. Paratexts are integral to many such behaviors, communicating information that gives players advantages in the game, or that allows them to subvert the game code. Paratexts also function as important vehicles for obtaining, transmitting, 
and maintaining gaming capital, often by communicating the information that facilitates cheating. Paratexts may therefore constitute an important control point over the disposition of gaming capital. Control over paratexts could allow a publisher to suppress or modify paratexts, either to prevent cheating, or to channel gaming capital to the advantage of the publisher.

Game developers therefore face a quandary: how to encourage development of paratexts in a fashion that will enhance the desirability of the game, making play attractive, but discouraging development detrimental to the business plan for the game. Certainly, publishers of other types of texts face something of this problem; popular television series and movies often leave a spume of commentary, fan fiction, and tributes in their wake. Some books, such as Twilight or The Lord of the Rings similarly inspire their audience to discuss, extend, and emulate the text. Communities coalesce around these works; they dress as the characters, hold conventions, learn to speak Klingon or High Elvish. There is undoubtedly a degree of "textual capital" sought and accrued by the fans of such works. The publishers of such texts must strike a balance between encouraging fan enthusiasm that will promote their business ends, and losing control of the paratexts that might generate additional revenues. More than a few copyright cases, including several that are pertinent to the analysis here, turn on such assertions of control.

However, gaming is somewhat unique in that players are invited and expected to interact with the text - playing the game is the point of the game. Relatively few of these other texts involve direct, rather than imagined, interaction. Game developers are therefore required to strike a balance few if any other publishers must negotiate: how to encourage actual manipulation of their text in a quantity and fashion that will further the developer's commercial plans, without creating incentives for textual manipulation, such as hacking, that would hamper those plans. Game developers, like other publishers, are keenly interested in the control of separate paratexts that spring up around their games, but must be additionally concerned over paratexts such as software and hardware devices, that directly alter the play of the game by altering the primary text they mediate. The ownership regime that allows developers to control their primary texts offers the possibility of controlling related paratexts.

\section{Copyright and Paratexts}

Game developers are beginning to assert copyright to control the use and abuse of the games that they publish. For example, Blizzard Entertainment, the developer of the World of Warcraft and other successful on-line multiplayer games, recently asserted copyright claims against the distributers of "Glider" software, a gaming robot or "bot that allowed players to automate their play, having their game characters function without the active control of the player. (MDY Services, LLC v. Blizzard Entertainment, Inc., 2008) Blizzard asserted 
inter alia that the operation of the Glider produced unauthorized copies of Blizzard software in the RAM of player computers. Blizzard has also clashed with game fans who attempting to sell their own World of Warcraft "how to" playing guides through eBay. (Public Citizen, 2005)

In some ways, the deployment of copyright to control game activity should not be surprising. From the very first days of arcade video gaming, copyright was asserted over the computer code that defined the game and over the audiovisual output of that code. Copyright claims followed video games to the home gaming console and the personal computer. (Samuelson, 1993) But along the way, a shift occurred: early gaming cases are directed toward commercial imitators and business rivals of game developers. A similar trend is apparent in the on-line gaming cases. Because copyright applies to certain fundamental uses of texts, and because texts inform human autonomy, copyright raises certain ethical and policy issues when it is asserted to control texts. These concerns are certainly present when copyright operates in the realm of commerce, but they become heightened when copyright is directed toward an individual's personal activities.

\subsection{THE SCOPE AND NATURE OF COPYRIGHT}

Copyright protects the original expression of an author, fixed in a tangible medium, in certain classes of works such as literary works, audiovisual works, sound recordings, musical compositions, dramatic works, graphic or sculptural works, and related types of artistic creations. (17 U.S.C. § 101) Oddly, most countries including the United States have chosen to extend copyright protection to computer software, despite the fact that it is not an artistic work, but a utilitarian work of engineering. The United States protects copyright as a "literary work," along with poetry, essays, and novels, apparently because it can be recorded as a symbolic notation that, in its source code format, can be read by humans.

Copyright confers the exclusive right of reproduction - the "copy right" which allows the holder to exclude others from making copies of the work. (17 U.S.C. § 103) But this right extends beyond literal copying to include exclusivity over copies that are substantially similar to the protected work. If the right of reproduction extended only to slavish copying, copies that reproduced the work word for word or pixel for pixel, it would be essentially meaningless: by making insubstantial changes, an infringer could evade the scope of such protection. Consequently, the scope of the right is broader than exact copies, including variations on the work where substantial copying, but not complete copying, has occurred.

In addition to the right of reproduction, copyright also confers a variety of other rights, including the exclusive rights of distribution, public display, and 
public performance. ${ }^{1}$ Perhaps most significant for this discussion is the exclusive right to prepare adaptations of the protected work, that is, to prepare derivative works based upon the copyrighted work. Classic examples of derivative works would include translations, sequels, and adaptations of stories to screenplay. The adaptation right has typically allowed control over derivatives that incorporate some portion of the initial work's original expression into the subsequent work. For example, the legislative history of the statute suggests that a commentary on a work would not be considered an adaptation, nor would an original work inspired by another - such as a musical composition inspired by a painting or novel. It is not enough to take an idea from the work; the work's expression of the idea must be incorporated into the new work for it to be adaptation.

\subsection{COPYRIGHT AND COMPUTER GAMING}

Copyright analysis of computer games is complicated by the various layers of copyrighted works entailed in the game. (Burk, 2006b) The most obvious and observable copyrighted work associated with the game is the output viewed on the screen: an audiovisual work incorporating animated images, sound, music, text, and increasingly, voice supplied by the players. This work might seem to be ephemeral; it changes constantly and for that matter differs for every player, depending on the choices they make in playing the game. Copyright law does requires fixation of a work for more than a transitory duration in order for rights to attach; however, some courts have indicated that this requirement is satisfied by the storage of computer code in memory, even temporarily.

Game outputs will frequently involve multiple overlapping and embedded copyrights. The audiovisual work generated by player interaction with the game is the subject of copyright, but is also likely comprised of elements that are individually the subject of their own copyright. Graphics depicting objects, background scenes, and non-player characters, as well as musical compositions, sound effects, and explanatory texts will each likely be the subject of discrete copyright for that particular creative element. Copyright in each of these works may belong to the developer, or may be licensed by the developer from another copyright owner.

In addition to the game outputs, copyright applies to the underlying software of the game, which generates the visual, auditory, and other interface output in response to the pre-set instructions of the developer and the input of the players. Additionally, the underlying game architecture will typically include some database of values representing the state of the game, including character status and other data objects manipulated by the players. This data

\footnotetext{
${ }^{1}$ In some countries, these rights would be incorporated into a more general right of communication to the public.
} 
may be difficult to distinguish from the gaming software, as the two must necessarily interact during play, and, since bits are bits, the distinction between software and data is never very pristine. In addition, the game software may include or draw upon more static databases that are unaltered by player activity, for example libraries of stock images, such as objects displayed by the game software during the course of the game.

Databases are not always subject to copyright, although databases can be the subject of copyright if they are sufficiently original, either in their subject matter, or in the selection and arrangement of the subject matter, or both. In copyright law, facts are typically not considered original, as they are assumed to exist in the state of the universe and do not originate from an act of authorship (Burk, 2007; Durham, 20001). Consequently the content of many databases comprising factual data, may fall outside copyright, as may and so the database as a whole. It is not altogether clear that the selection and arrangement of the game values database constitutes original expression, as it is comprised of values generated by game play, but not necessarily explicitly selected by players. Certainly the values do not constitute facts or measurements drawn from the external world, although their selection is also not entirely arbitrary, as they must represent persistent characteristics of the game. The data set comprising the game may be subject to copyright, but also itself gives rise to other copyrightable output.

\subsection{SUBSTANTIAL SIMILARITY AND DERIVATIVE WORKS}

Thus, copyright applies to the set of creative works that constitute the computer game itself. But numerous of copyright cases suggest that the ability of copyright owners to control related paratexts may also be quite broad, across a range of paratexts in print and digital media. For example, in Castle Rock Entertainment, Inc. v. Carol Publishing Group the creators of the television situation comedy series Seinfeld sued the publishers of the "Seinfeld Aptitude Test," or "SAT," a book comprising a set of quizzes about the characters, events, and internal details of the television program. (Castle Rock Entertainment, Inc. v. Carol Publishing Group , 1998) The book posed readers with a series of trivia questions such as, "What candy does [the character] Kramer snack on while observing a surgical procedure from an operating-room balcony?" 2 The producers of the show prevailed on a theory that the book publishers had impermissibly copied original expression from the show. The court held that the series of questions in the SAT incorporated original expression from the show and so were "substantially similar" to the "Seinfeld" program.

\footnotetext{
${ }^{2}$ For inquiring minds who may want to know, the correct answer is "Junior Mints."
} 
What is striking about this holding is that the SAT is clearly not a substitute for any aspect of the "Seinfeld" television program. The test certainly is not a "copy" of any existing episode of the television show, nor does it offer any new stories or representations of the characters, plots, settings, or premises of the show. It is not a new episode of Seinfeld, neither is it a novel, play, poem, or other narrative work based on the elements of the show. It is a text about the show, rather than an extension of the "secondary world" created by the narrative structure of the television program. (Tolkien,1947) To the extent that the Test "copied" elements of the show, it did so in a disaggregated form, taking discrete occurrences from the program's narratives and recasting them as multiple choice questions.

As a text about the show, the "Seinfeld Aptitude Test" is a prime example of "paratext" in the sense that Consalvo has used the term. Although the Seinfeld television program is not a computer game, it constitutes a text in the broader sense in which Consalvo uses that term. The text of the "SAT" is related to the main text of the television episodes, taking the television episodes as canonical. The book was directed to fans of the show, who have acquired, enjoy, and perhaps wish to demonstrate detailed knowledge of the show: we might call this "show capital" paralleling in many respects Consalvo's "gaming capital." The book's quiz helps define a community of fans for the show, promotes an ongoing interest in the show, and imparts information about the internal situations and characters of the show.

Creating paratexts requires some degree of copying from the initial text; the paratexts can only refer to the text by appropriating enough of its characteristics to evoke a relationship between the two. The United States Supreme Court has recognized this relationship in the context of one type of paratext, the parody, where the court has held that it is fair to copy enough of the initial text's material to "conjure up the original." But clearly any type of paratext will need to refer and point to the initial text in order to establish its relationship to that text. The ability of copyright holders to exclude paratexts as "substantially similar to" or "derivative of" the text they modify extends the control of the copyright owner over a wide range of "gatekeeping" texts that mediate the reader's experience with the primary text.

Neither does the SAT present an unusual case. Much the same set of issues was recently litigated with regard to the "Harry Potter Lexicon," an encyclopedic collection of regarding the plots, characters, and milieu of the popular "Harry Potter" fantasy novels. Fans of the books assembled information drawn from the novels into an encyclopedic form that was published on the Internet. The author of the Potter books, J.K. Rowling, allowed and even approved of the on-line version of the Lexicon, but filed suit to prevent the coordinator of the Lexicon from publishing it in printed book format. As in the Castle Rock case, the court hearing Rowling's complaint held 
that the re-formatting of material from the Potter books into encyclopedic form produced a "substantially similar" work that infringed the books, effectively giving Rowling the right to control rearrangements of facts drawn from her stories.

\subsection{COPYRIGHT IN VIDEO GAMES}

The doctrines of substantial similarity and adaptation have been extended to computer media, including computer games. For example, Consalvo discusses the holding in Lewis Galoob Toys, Inc. v. Nintendo of America as a key case for computer gaming. (Lewis Galoob Toys, Inc. v. Nintendo of America, 1997) The Lewis Galoob case concerned the "Game Genie," a device designed for use with the Sony PlayStation game console. When attached to the console, the Game Genie would speed up game play and increase the abilities of the characters controlled by the player, making play more interesting than, or at least quite different from the game as distributed by the manufacturer. The changes in play were accomplished by alter the output of the PlayStation console, changing the value of a particular parameter that was communicated to the television displaying the game in progress. Only the output value, and not the underlying game software was altered by the device; when the Game Genie was removed, the console would play as specified by the manufacturer. This was the key factor in the decision reached by the Ninth Circuit court of appeals. The court held in favor of the Game Genie manufacturer because of the device to did not permanently alter the underlying copyrighted work; since there was no permanent change to the Nintendo software or its output, the court concluded that no derivative work had been created.

Consalvo briefly touches on the debate regarding the Game Genie, rightly identifying it as a key decision, and concluding that the Ninth Circuit's opinion declared such devices to be legal, paving the way for other such game modification devices. While this may have been the practical effect, the case has broader implications for the allocation of gaming capital and the related development of game paratexts. The issue is not so much the legality or illegality of game altering devices, it is the broader issue of the circumstances under which such devices can or cannot be prohibited. Taken at face value, the holding of the case suggests that if game cheaters have not altered the underlying code of the game, that no claim would lie regarding the creation of an unauthorized derivative work.

This simplest reading of the case could have broad ramifications. Many on-line games are structured around a client-server model, whereby the game interface is generated from a local application on the player's computer, but updates to the status of the display are exchanged with, and stored upon, a remote server. Many on-line game hacks are based upon exploiting this architecture, intervening in the communications protocols between client and 
server to altering the values of data exchanged between the two. This allows the hack to change the game and character updates, giving characters more experience or greater ability, generating more monsters faster, or immobilizing them, or conferring other advantages. One reading of the Galoob case might be that if only the values exchanged between server and client are changed, and not the underlying gaming program itself, then no derivative work has been created and no copyright claim of that type could be asserted by the developer against the hacker.

At the same time, it is difficult to know precisely how this analysis might play out in the context of on-line gaming, even for hacks exploiting the clientserver architecture I have described. The outcome of the analysis depends to some extent on whether consideration of derivative works stops at the gaming software proper, or extends to the database created by the operation of that software. To take the example of the client-server hack, even though the hack may not change the underlying software, but only changes the output of the software, those altered outputs are likely stored somewhere - the enhanced experience, or skills, or game currency acquired by a character through a particular hack is likely stored on the game server. After all, the point of the hack is usually to gain some advantage that will be permanently reflected in the avatar's status within the game. Neither is the stored data limited to the particular character; its relationship to other parts of the game - in tasks or quests completed, transactions engaged in, objects acquired - is also permanently changed.

The result of any given hack is that value parameters throughout the game database are altered. If we consider the database of game values, rather than the software game engine, as the copyrighted work in question, then the reasoning of Galoob might lead us to believe that an unauthorized derivative work has been created by the hack: the database has been altered and those alterations are stored and fixed. Of course, any player of the game makes alterations to the database values in the course of play - every player creates or contributes to the database. For massively multiplayer games, the game database is in effect a massive derivative work created by the collaboration of multiple players. This line of reasoning therefore shifts our attention to whether that work can be somehow tainted, rendered illegitimate, by the activity of hacks and cheats.

\subsubsection{Narrative Code}

The particular holding of the Lewis Galoob case - that altered game output is permissible so long as the alterations are not fixed -- stands in some doubt, as it is seemingly at odds with more recent decisions in both the Ninth Circuit and elsewhere. Key among these cases is the decision in Micro Star v. FormGen, a case involving the PC game "Duke Nukem in 3-D." (Micro Star v. FormGen, 1998) The game developer, FormGen, made available to its users the tools to 
develop alternate game levels and encouraged the sharing of such user-created game files on its web site. The advanced or alternate game versions existed as "MAP" files, or sets of game instructions, that could draw upon a graphic library of character and object images provided with the game itself, but which would sequence, arrange, and display the library images in such a way as to provide a more challenging game experience. Thus, the MAP files operated together with other components with the Duke Nukem game, but could not themselves independently generate game output, lacking the necessary content to do so.

The defendant in the case, Micro Star, assembled on compact disc collections of the user-created files, and marketed the disc without either the authorization of FormGen or of the users who developed the files. FormGen claimed that the taken MAP files constituted derivative works that contained protected expression, giving them standing to sue. In return, Micro Star argued that the copied and distributed MAP files failed the requirements of a derivative work; that they incorporated no protected expression and did not constitute any concrete or permanent alteration to the FormGen's original expression in the game.

The court held in favor of FormGen, reasoning that a description of a derivative work is equivalent to a derivative work. The court further held that the MAP files incorporated protected expression as a sort of narrative; because the MAP files dictated the placement and sequence of the Duke Nukem characters, the MAP files therefore constituted a "story" about the FormGen game characters. The opinion reasons that the alternate game levels were derivative works of FormGen's Duke Nukem story, and that by "describing” the placement and arrangement of the graphics in those derivative works, the MAP files constituted in effect the plot, the sequence, the narrative of those derivative works.

It is difficult to see what aspects of the Duke Nukem game were incorporated into the MAP files that were illicitly distributed by Micro Star. The files comprised instructions for calling up, arranging, and displaying other files from the program graphics library. The opinion suggests that sequencing and arranging such elements constitutes a type of narrative, a new story about Duke Nukem and the post-holocaust world he inhabits. But the MAP files contained no distinctive "story" regarding Duke Nukem unless paired with the content of the Formgen graphics library, and even assuming that this analogy is credible, copyright has never extended to the general plot of stories or other narrative works, only to the particular expression of a given story. Nonetheless, the case suggests contrary to Lewis Galoob that instructions for the creation of derivative works may infringe a game's copyright, even if the game is not permanently altered. 
The MAP files that the MicroStar opinion characterized a sort of narrative produced by players with the authorization of the game developer. Such derivative works for PC games presage certain copyright issues that arise in online role playing games. In previous work I have discussed the difficulty of classifying avatars and their game narratives under the categories recognized in the Copyright Act. (Burk, 2006b; Burk, 2008a ) Game developers have asserted copyright over the characters and narratives created by players incident to the game. There argument is that players merely draw upon choices provided by the developer. This account of copyright in the game probably understates the contribution of the players to the work that is generated. I have indicated that the developer likely holds copyright in individual elements of the game, and be said to have selected the elements from which players choose, the options for play are extensive and elements of the game are arranged in fairly complex ways by the players. The number of combinations is sufficiently large, and the number of possible play outcomes sufficiently diverse, that it seems likely players have added original expression to develop the audiovisual output of the games - indeed, in a multiplayer setting, many players have collaborated to generate the state of the game at any given time.

Given the contribution of the players to the game output, and assuming that the players are authorized to engage with the game's copyrighted material, the end product seems likely to be a derivative work, or even a work of joint authorship. Works are the product of joint authorship if two or more contributors combine original expression intending to create a single, unified work. It is somewhat unclear whether this is the case for game output; certainly a game producer must contemplate that players will play the game, and so arrange game elements to perform the audiovisual output. But it may be less certain that the game developer considered the activities of the players as original authorship, or intended the production of an audiovisual work as such. If the game developer's purpose is simply to provide entertainment, or to make money, the intent needed for a joint work might not be present.

That leaves the possibility that the game output is an authorized adaptation, a derivative work. (Burk, 2006b) But if the players are not authorized to engage with the game's copyrighted material, then what might have been a derivative work or work of joint authorship becomes infringement; an adaptation in violation of the copyright holder's exclusive rights. In the course of normal game playing, one would expect the player-generated output to be authorized: generating an entertaining output is the point of playing, and the developer both expects and facilitates this outcome when making the game available. However, players manipulating the game's elements beyond the authorization given by the game developer may be creating an adaptation without the approval of the copyright holder. 
The distinction between authorized collaborative works and unauthorized collaborative works raises the question as to what scope of authorization is granted in playing the game; what activities by players might the developer be expected to have anticipated and acquiesced in? The answer to this question will be contractual rather than statutory: what was the agreement between developer and player allowing the player to manipulate the copyrighted material of the game? In the absence of an explicit agreement, we might be forced to infer what player activity is likely to fall within or without the parties' expectations. But there is routinely at least an attempt to state explicit terms to this agreement. At log-in, games routinely require a manifestation of agreement with terms of service (ToS) that attempt to spell out what actions are permissible and which aren't. Much of the authorization question may depend on the ToS that the players agree to when initially signing on to the game. Hacking or game play in excess of the authorization given in the ToS might throw the output of play into the realm of infringement rather than a derivative work; in fact, violation of Blizzard's ToS was the explicit basis for the copyright claim in the "Glider" software law suit.

\section{Ethical Implications}

Digital texts highlight the interactions between the copyright in primary texts and the control of paratexts. The malleability of bits erodes the distinction not only between discrete texts, but between author and consumer. This effect is apparent in the action of on-line gaming, where players and developers virtually collaborate to generate unique narratives that are structured as vast databases but displayed as audiovisual works. The game constitutes a series of texts, rather than a single text. The audiovisual output proceeds differently depending upon the choices made by players; in the case of multiplayer games, every player will see a different, related but unique game variant depending upon viewpoint. All texts share this character to some extent, as different readers interpret and "recode" the symbols inscribed by the initial author. (Foster, 1985; Rotstein, 1992) My experience reading Wuthering Heights is not quite anyone else's. Such recoding becomes particularly obvious in the case of digital works, which are often amenable to, or specifically designed for, user interactivity, and may allow readers to quite literally recode the text, transferring their interpretation from carbon memory to silicon memory. (Jaszi, 1992; Chon, 196; Burk, 2006a).

\subsection{WRITERS AND READERS}

I have argued elsewhere that for these reasons copyright may be particularly ill suited to digital media, as it assumes a discrete and static model, not only for 
texts, but for interaction with texts. (Burk, 2006b). Copyright assumes that authors produce texts and readers consume them. The distribution of rights in the copyright system is disrupted when readers interact with a text as both creators and consumer. Similarly, the copyright system has little latitude for cocreated works; it classifies creators as independent authors, joint authors, compilers, or infringers. Any textual reformulation that fails to fit the narrow confines of the former two legitimated categories are thrust into the last, illegitimate category - one reason that the system is so inimical to the digital culture of sampling, remix, and mash-up. (Lessig, 2008)

Some of the ethical implications of copyright in this milieu are familiar. First, as I have observed elsewhere, there is the matter of ownership in a setting where gamers may invest a significant degree of personal capital in their avatars. (Burk, 2006b) Intellectual property is typically justified as an economic incentive to creation, as a reward for labor, or as the investment of personality in a creative product. These justifications are typically marshaled in favor of ownership for product developers. But in a game setting, the players may not only put considerable effort into creating on-line characters, they may strongly identify with them on an individual level, as representative of their own personality. Thus vesting copyright ownership exclusively with game developers carries negative implications for player autonomy and individuality.

Second, aside from the personal investment of players in the game, copyright enforcement may prevent certain types of interactions with the text, and indeed within the text, as players participate in the development of characters, narratives, and audiovisual displays from within the fabric of the game they are playing. This may occur directly by prohibiting certain variations of game play. Or it may occur indirectly as copyright is applied to paratexts, by suppressing or altering of the texts that would mediate reader interaction with the primary text. Assertion of copyright ownership permits the production of paratexts only if they are "authorized" in every sense of that word: both in the sense of having been approved of by the copyright holder and in the sense of having concomitantly been converted to the product of an author.

These constraints clearly affect a wide range of paratexts, in greater or lesser proximity to the primary text. As copyright was classically formulated in print media. the farther removed from the primary text the paratext becomes, the more attenuated copyright claims should be, and the greater the interest in fostering original expression should be. But as digital media facilitates the collapse of text and paratext, the features that indicated such distinctions have become indistinguishable. The cases reviewed here indicate that copyright already extends a considerable distance along the spectrum of paratexts. As the connection between text and paratext becomes increasingly seamless, the extension of copyright control to previously discrete paratexts is likely to increase. 


\subsection{POLICING PARATEXTS}

The spectrum of paratexts related to a primary text also implicates a continuum differing interests in the production of such paratexts. For example, as constraining as they may be, many of the technical limits built into gaming technology are simply a part of the structure of the game itself - the enjoyment of the game may be bound up in such limitations. Violation of the game structure carries with it consequences beyond the interests of the violator and the game developer, impacting the autonomy and enjoyment of other players. Curtailing the circulation of software scripts that allow subversion of the game code may be essential to prevent disruptions of the game that would be detrimental to the majority of players. To a lesser extent, revealing game codes, hidden features, or playing strategies can also have a detrimental effect on the enjoyment of other players, either by depriving them of the challenge of discovering such features themselves, or by discouraging those who may not have access to such information, but who see other players effortlessly surpassing their own level of achievement.

Invoking legal rules against certain types of paratexts and certain methods of obtaining gaming capital may therefore protect the interests of the gaming community as a whole, admittedly at the expense of individual autonomy within the game. Copyright may be seen as a tool for protecting the integrity of the game, and the personal investment of other players, quite apart from the financial interest of the game developer. Curtailing some degree of playing freedom via intellectual property enforcement may be seen as balancing individual preferences against the interests and enjoyment of the larger body of players. This is a familiar balance. In a general social context, it is fairly well accepted that regulation can and should be deployed to constrain anti-social behavior in the public interest. Such laws certainly will have individual costs for the autonomy of a certain number of people, but on balance the cost is seen as justified by the overall benefit to multiple interests, including autonomy, that result from securing social order.

In such instances, the interests of the game developer may to some extent coincide with those of players. But suppression of anti-social readings of the game is by no means likely to be motivated by the interests of the players, nor necessarily by those of the gaming community. The beneficial effect to the gaming community may be a happy but uneasy coincidence. It is questionable how far the two sets of interests travel together. And, the calculus of personal cost and public benefit may change when legal constraints are applied outside of game play, to deter activity that does not constitute the alteration of the actual game structure. Copyright restriction of paratexts such as fanzines, websites, or advice manuals places control over much of the commentary and discussion about the game into the hands of the party with an economic interest 
in the promotion of the game. That interest will not necessarily favor robust debate and criticism regarding the game.

To be certain, the utilitarian calculus for the policing in-game behavior not altogether divorced from the issue of policing paratexts. Texts are to some extent shared enterprises in the sense that culture is a shared enterprise, and at some level the individual interpretation of texts may have an impact on others within the shared cultural network. The logic of the greater good can work both ways: some commentators have argued that there exists a general social interest in preserving a stable reading of copyrighted texts; that the individual interest in reinterpreting or re-coding texts is outweighed by a larger societal interest in preserving a canonical reading, and that copyright preserves such canonical readings. (Hughes, 1999).

Yet the vast weight of commentary and authority on copyright and recoding has tended to focus instead on the value individual freedom, expression, and human rights. Democratic societies are in general somewhat suspicious of giving interpretive control into the hands of a single self-serving entity, hence the primacy of rights to expression in national constitutions and international treaties. A variety of scholars have recognized a general tension between freedom of speech and control of speech via copyright; the United States Supreme Court has justified restraint of speech via copyright as a mechanism that will promote production of expressive works, a social gain that hopefully outweighs whatever expressive losses that accompany the deployment of copyright. (Campbell, ; Lessig, 2004) For the same reason, copyright is rife with exceptions, including a general provision for "fair use," that foster some degree of interpretive recoding of copyrighted texts is critical to personal expression.

These concerns taken in the aggregate, translate into a set of broader societal concerns over human flourishing. Chief among these is a concern over the control of interpretation and meaning of socially shared texts. This problem in copyright remains relatively unexplored, except as an adjunct to the tension between freedom of speech and legal suppression of texts. (Vaidhyanathan, 2004) But beyond concern over the production or suppression of expression lies a broader concern that exclusive rights confer a substantial degree of control over the meaning of the primary text. Suppressing liminal texts that mediate interactions with the primary text is troubling for its own sake, but also for the impact on a reader's understanding of the primary text. The reader's understanding is shaped by enforcement of the copyright holder's preferred interpretation of the owned primary text. If game scenarios are regarded as a text, copyright and related legal regimes are enforcing certain readings of that text, and forbidding other interpretations -- a constraint on autonomy and expression that reaches far beyond the activity of "cheaters," however defined. 


\section{Conclusion}

Gaming, as I said at the beginning of this chapter, is about the rules: who makes the rules, who breaks the rules, how and why and when the rules are enforced. This is to say that gaming, like ethics and like law, is about power relations. By conferring control over paratexts, copyright and related legal claims endow the developers of on-line games with significant panoply of mechanisms to control the acquisition and disposition of gaming capital. But control over paratexts also entails control over the manner in which gamers experience and interpret the games they are playing, as well as how they experience the communities that grow within and around such games. Thus the coercive power of copyright may confer on game developers an unintended scope of control, not merely to define not only the rules that apply within their games, but to establish rules and norms beyond the game as well. The manipulation and suppression of paratext by means of copyright is certainly not a concern limited to the context of gaming, but is readily apparent in the context of computer games, and we can begin to consider broader social questions of information control through careful consideration of copyright and paratext in computer gaming.

\section{References}

Baym, N. K. (1993). Interpreting Soap Operas and Creating Community: Inside a ComputerMediated Fan Culture. Journal of Folklore Research, 30, 143-177.

Blizzard Entertainment, Inc. v. In Game Dollar, LLC, SACV07-0589 JVS (C.D. Cal. Jan. 28, 2008).

Burk, D.L. (2008a). Information Ethics and the Law of Data Representations. Ethics \& Information Technology 10:135-147.

Burk, D.L. (2008b). The Mereology of Digital Copyright. Fordham Intellectual Property Media \& Entertainment Law Journal 18, 711-739 (2008).

Burk, D.L. (2007). Method and Madness in Copyright Law, Utah Law Review, 2007, $587-$.

Burk, D.L. (2006a). Copyright and Feminism in Digital Media, American University Journal of Gender, Social Policy \& Law, 14:519-549.

Burk, D.L. (2006b). Electronic Gaming and the Ethics of Information Ownership. International Review of Information Ethics 4 http://www.i-r-i-e.net/inhalt/004/burk.pdf

Burk, D.L. \& Gillespie, T. (2006). Autonomy and Morality in DRM and Anti-Circumvention Law, Triple C: Cognition, Communication, Cooperation, 4:239-245.

Castle Rock Entertainment Inc. v. Carol Publishing Group, 150 F.3d 132 (2nd Cir. 1998).

Chander, A. \& Sunder, M. (2007). Everyone's a Superhero: A Cultural Theory of 'Mary Sue' Fan Fiction as Fair Use, California Law Review 95, 597.

Chon, M. (1996). New Wine Bursting from Old Bottles: Collaborative Internet Art, Joint Works, and Entrepreneurship, Oregon Law Review 75, 257-276.

Consalvo, M. (2007) Cheating: Gaining Advantage in Videogames (MIT Press) 
Consalvo, M. (2003) Cyber-Slaying Media Fans: Code, Digital Poaching, and Corporate Control of the Internet. Journal of Communication Inquiry, 27, 67-86.

Durham, A. (2001) Speaking of the World: Fact, Opinion, and the Originality. Standard in Copyright, Arizona State Law Journal, 33, 791 - .

Foster, H. (1985). Recodings: Art, Spectacle, Cultural Politics. Seattle: Bay Press

Hughes, J. (1999). Recoding Intellectual Property and Overlooked Audience Interest, 77 Texas Law Review 77:923-1010.

Jaszi, P. (1992). On the Author Effect: Contemporary Copyright and Collective Creativity, Cardozo Arts \& Entertainment Law Journal, 10, 293- .

Lessig, L. (1999) Code and Other Laws of Cyberspace New York: Basic Books.

Lessig, L. (2004) Free Culture: How Big Media Uses Technology and the Law to Lock Down Culture and Control Creativity, New York: Penguin Press.

Lessig, L. (2008). Remix: Making Art and Commerce Thrive in the Hybrid Economy New York: Penguin Press.

Lewis Galoob Toys, Inc. v. Nintendo of America, Inc., 964 F.2d 965 (9th Cir. 1992).

Lunenfeld, P. (1999) Unfinished Businesss. In P. Lunenfeld (ed.) The Digital Dialectic: New Essays on New Media 6-23 Boston: MIT Press.

Liu, J. (2003). Copyright Law's Theory of the Consumer, Boston College Law Review 44, 397431.

MDY Services, LLC v. Blizzard Entertainment, Inc., No. CV06-02555-PHX-DGC (D. Ariz., Sept. 29, 2008).

Microstar v. Formgen, Inc., 154 F.3d 1107 (9th Cir. 1998).

Public Citizen, In Settlement Victory, Software Company Allows eBay Sale of Guide to Popular Video Game (June 9, 2005) http://www.citizen.org/pressroom/release.cfm?ID=2217

Reidenberg, J. (1998) Lex Informatica: The Formulation of Information Policy Rules Through Technology. Texas Law Review 76:553-584.

Rotstein, R.H. (1992). Beyond Metaphor: Copyright Infringement and the Fiction of the Work, Chicago-Kent Law Review 68, 725 - .

Samuelson, P. (1993). Fair Use For Computer Programs and Other Copyrightable Works in Digital Form: The Implications of Sony, Galoob and Sega, Journal of Intellectual. Property. Law, 1, 49- .

Tolkien, J.R.R. On Fairy Stories Essays Presented to Charles Williams, Oxford University Press C.S. Lewis ed. 1947 38-89

Tushnet, R. (2007). Copyright Law, Fan Practices, and the Rights of the Author. In J. Gray, C. Sandvoss, \& C.L. Harrington (eds.) Fandom: Identities and Communities in a Mediated World 60-71, New York: New York University Press.

Vaidhyanathan, S. (2004) The Anarchist in the Library: how the clash between freedom and control is hacking the real world and crashing the system. New York: Basic Books. 